\title{
Collaborative Game Environments for Informal Science Education: DinoQuest and DinoQuest Online
}

\author{
Walt Scacchi and Robert Nideffer \\ Game Culture and Technology Laboratory \\ University of California, Irvine \\ Irvine, CA 92697 \\ \{wscacchi, nideffer\}@uci.edu
}

\author{
Joe Adams \\ Discovery Science Center \\ Santa Ana, CA \\ 2500 N. Main St. Santa Ana, CA \\ 714-542-2823
}

\begin{abstract}
We describe concepts and results that arose from the development and deployment of a large-scale collaborative game environment called DinoQuest and DinoQuest Online. As an interoperable game environment, DQ and DQO provide a unique experience and approach to informal science education. DQ and DQO are games for helping school-age children to learn about science (or more specifically, life science and dinosaurs). In this paper, we identify and examine different collaborative group forms that emerged to play DQ and DQO, as well as the affordances that help facilitate collaborative game play. Along the way we provide examples of the collaborative groups, affordances, and game play from DQ and DQO.
\end{abstract}

\section{Introduction}

We have developed a large-scale collaborative game environment deployed in a regional science center that joins physical and online activities in the domain of informal science education. The Discovery Science Center (DSC), located in Santa Ana, $\mathrm{CA}^{1}$, is a regional science center that families and school groups visit in order to experience a diverse variety of interactive science exhibits. These exhibits bring scientific subjects or concepts to life in a hands-on, fun, and entertaining manner. DSC focuses on interactive exhibits as opposed to passive exhibits of scientific artifacts as might be found in a museum that memorializes the history of scientific concepts, scientists, and inventions. DSC is also situated in municipal region, Orange County, CA, whose population spans large concentrations of ethnic immigrants (from Mexico and Latin America, Asia, Middle East, etc.), as well as very affluent to very poor communities. During 2005, more than 275,000 people engaged in DSC visits or outreach activities, while in 2007 more than 425,000 people were engaged, including 150,000 K-12 students of which nearly 90,000 participated in school group visits to DSC. Thus, DSC [2008] exhibits and educational outreach programs are tailored to meet the interests of different communities, age groups, school educators, and other constituencies.

In 2004, effort began at DSC to develop a new interactive game-based exhibit that would focus on dinosaurs as the basis for introducing, demonstrating, and engaging visitors with the concepts from life science (e.g., skeletal systems, elements, and function; digestive system; prey-predator relationships).

The life science concepts selected for presentation in the exhibit were those that correspond to curricular topics found in K-6 grade science education standards for California, which are nearly identical to the National Science Education Standards ${ }^{2}$. The UCI Game Culture and Technology

1See, for example, http://www.answers.com/topic/santa-ana?cat=travel

2 http://www.nap.edu/readingroom/books/nses/overview.html. These standards are not part of recent U.S. Federal initiatives like "No Child Left Behind" nor are they the basis for testing scientific knowledge by school grade. Instead, they focus on 
Laboratory [UCGameLab 2008] was invited to join this project at this time, and project went into design and operational planning in early 2005. This exhibit was designed to enable the development and deployment of both a physical game-based interactive exhibit at the DSC that would be linked and integrated with a Web-based online game environment. The physical exhibit called DinoQuest (DQ) became operational in mid 2006, while the online game environment called DinoQuest Online (DQO), went into full-scale operation in 2007. Both DQ and DQO were conceived, designed, and deployed as collaborative science learning game (SLG) environments, and can be evaluated as such. The remainder of this paper focuses on examining and explaining DQ and DQO as collaborative SLG environments, as well as what facilitates different forms of collaboration and collaborative game play.

\section{Informal Science Education through Science Learning Games}

Informal Science Education (ISE) is concerned with providing and experiencing scientific concepts, methods, and devices drawn from different science disciplines in settings outside of school, where formal science education occurs. Science centers, museums, after school clubs, and public media (e.g., the Nova television series broadcast in the U.S over the Public Broadcasting System/PBS) are the common settings for ISE, though ISE can also occur at home in settings with family or friends. What is key to ISE is that it is elective, discretionary, and a matter of free choice in terms of the content provider, as opposed to schools whose choices may be determined by school boards or others. However, in our view, science centers that showcase interactive, hands-on exhibits are an ideal setting to deploy SLGs, as part of an overall environment for ISE that is readily accessible to a large public audience.

SLGs are games first and foremost. They are conceived, designed, played, and analyzed as computer games, rather than educational courseware, simulations, or interactive presentations of science concepts [cf. WDIL 2008]. As such, criteria such as intrinsic motivation, effective game play balance, and fun [Malone and Lepper 1987, Koster 2004, Salen and Zimmerman 2003] were among our requirements for DQ and DQO, as were other criteria on computer games as art, culture, and open source development practices that we have been investigating [e.g., LaFarge and Nideffer, 2002, Nideffer 2002, Scacchi 2004].

SLGs are a small and mostly marginalized genre of computer games when one looks at the international computer game industry. No companies appear to be making millions of dollars from their best-selling SLGs. In fact, most of the large, well-known computer game companies avoid developing games that are envisioned as "educational" and targeted to specific age-skill groups. Instead, they more often seek to develop games that are fun, entertaining, and engaging, as well as focused on fantasy worlds, rather than on education and academic subjects. Subsequently, there is comparatively little industry interest in developing and deploying educational games in general, and SLGs in particular. However, as some game scholars and educational theorists have observed, many computer games succeed because they are great learning environments that embody both classic and modern theories of constructivist learning, self-identity through role play, reflective thinking, domain-specific specialist language skills, and multi-player socialization [Bainbridge 2007, Gee 2003, Shaffer 2006]. Thus, we see the emergence of the so-called "serious games" community of small, independent game

identifying for teachers, parents, and others what scientific concepts and practices students should be taught and learned (hopefully) in order to become scientifically literate citizens through their K-12 education. Students who excel or become enthusiastic learners of such materials may then be prepared for college level study and a career in a science, technology, engineering, or mathematical field. 
development studios and academic/industrial research groups that are beginning to invest resources into development of SLGs. But serious games need not be fun [Bogost 2007] nor collaborative to be effective. In contrast, our choice was to develop and deploy SLGs that would be both fun, collaborative, and oriented to free-choice science learning, which is generally a core requirement for interactive exhibits deployed in a regional science center [cf. Allison-Bunnell and Schaller 2005].

\section{Collaboration through Games and Gameplay}

Many games focus on single players, while others seek to encourage multi-player game play. Learning and educational experiences are also similarly focused on individual students or small groups. But where and how does collaboration or collaborative game play fit into such engagements?

Massively multi-player online games (MMOGs) are widely recognized for fostering modes of collaborative multi-player game play as one of their core features and modalities of experience. However, collaboration, in the form of multi-player online discourse and social interaction, often happens around the game to enable collaboration within the game [Nardi and Harris 2006]. Thus, reading, communicating, and interacting with others through multiple media/modalities [cf. Rieber 2005] become a capability for enabling distributed cognition and learning, both through and around game play, whether online or offline. Based on our observation and development of interactive exhibits at science centers, museums, and other settings, we have found the following kinds of collaborative groups are commonplace:

\section{Collaborative group forms}

Family group - a family is a problem-solving group that can involve members of different age groups that span generations (children, siblings, parents, grandparents, relatives, and others). Family groups may act in a hierarchical manner when some members (e.g., parents who can read) direct the activities of other members (children who cannot yet read) based on prior family arrangements or skill.

School classroom group - a school classroom group may engage in collective problem-solving when teachers or teaching assistants direct the attention of a classroom group (students) to focus on individual, collective, or competitive problem-solving tasks in order to reveal their comprehension (or lack thereof) of the problem at hand, and to propose a solution to it.

Inter-family group - a multi-family or inter-family group involves members from two or more family units that typically do not have a prior hierarchical relation between them, so that any joint action between families must be negotiated in some manner in order to proceed and benefit one or more of the participating families.

Mixed reality group - an mixed reality group consists of people in a physical setting who interact with other people seen or engaged online through their multi-media avatars (online representations or "ghosts" of the individual people participating/rendered online only). The avatars may be under live, real-time control by remote persons, or may be pre-recorded audio-video sequences controlled through online agents whose actions or behaviors are scripted in advance to respond to user input events.

Online multi-player group - an online multi-player group arises when all persons participating in a group act individually or collectively through online characters, and these characters may shown with human-like or animal-like appearances. 
Project development group - the group of people from often different organizations who are brought together for the purpose of conceiving, designing, producing, deploying, operating, and sustaining an interactive exhibit or shared computing application.

However, the emergence of these different forms of collaborative game play groups is not simply spontaneous. Instead, their emergence is facilitated by a number of socio-technical affordances for what to do, and how to such things alone or with others [Anderson and Sharrock 1993, O'Day, Boborow, Bobrow, and Shirley 1998] that not only situate a hands-on interactive science exhibit or SLG, but help make such collaboration fun, engaging, playful, and entertaining [cf. Muramatsu and Ackerman 1998].

\section{Affordances for game-based collaboration in groups}

Venue - part of what enables small groups to interact, communicate, and collaborate is the venue or place where they meet. Places like a science center convey a set of expectations to many visitors about what this place is about (e.g., observing, touching, or interacting with hands-on exhibits, and being amused along the way), and what kinds of individual or group activities are accommodated (talking, adults guiding the activities of children with them, seeing other people interacting with other exhibits to help learn how to interact or experience an exhibit, etc.). Science centers are places people freely choose to visit, typically as a family unit. ${ }^{3}$

Game genre - a collaborative game is generally a multi-player game or game genre (e.g., adventure/quest game, role playing game, first-person shooter/action game) that can be easily recognized by potential players who may already "know" how to play or what to do as a team, and thus can quickly learn how to start playing the game at hand.

Game exhibit and content - a game exhibit entails both the means and ways for engaging and experiencing the game while going through the game's content. The means are the form of the exhibit - its devices, apparatus, mechanisms, etc. - while the ways are navigational or interactional cues provided to players in order to move through the game's work/play flow.

Game infrastructure - behind the scenes of an interactive game-based exhibit is some arrangement of information technology (hardware and software) and telecommunications (networking) systems that enables the ongoing operation or use of the game by large numbers of players over different periods of time.

Game play situation and participants - each game provides a context and experience for its players, who may differ in their skill, mastery, or interest in the game. Part of the context and experience is conveyed through the game play activities and tasks that players are expected to perform in order to advance or make progress towards completion of the game (at least in games that are goal-directed).

Thus, another requirement for developing and deploying SLGs at a science center is to support such a variety of collaborative group forms and affordances. Now we turn to provide examples of the collaborative groups and affordances that emerged through DQ and DQO.

\section{DinoQuest and DinoQuest Online}

Collaborative groups, affordances, and game play for DQ and DQO can be seen through a number of

3 DSC does not admit unsupervised children under the age of 16 . Thus children come with their parents, older siblings, other family members, responsible adults, or school classroom groups. 
examples. The examples that follow are organized and presented first for DQ, second for DQO, and last for DQ and DQO jointly. Similarly, these examples help document where and under what conditions collaborative game play can be easily observed by outsiders, and engaged by insiders (DQ and DQO game players).

\section{DinoQuest (DQ)}

The DinoQuest venue - The DQ venue occupies more than 30,000 square feet of outdoor exhibit space at the DSC. It cost more than \$6M and two years to develop. There are 13 life size dinosaur models, including a partially articulated Argentinosaurus (one of the largest known dinosaurs at over 120' in length), T-Rex (a complete skeletal replica of "Stan BHI 3033" at the Black Hills Institute for Geologic Research), triceratops, smaller raptors, and so on, all based on paleontology discoveries in the late $20^{\text {th }}$ Century. The Argentinosaurus was designed around a bridge so that visitors can walk through it and physically interact with simplified renditions of some of its internal organs ${ }^{4}$. DQ is partially visible by automobiles driving by the DSC, including those of the adjacent Interstate 5 freeway. DSC also routinely advertises its featured exhibits in the print and electronic news media, which all helps to develop awareness, interest, and attendance to DSC and its featured exhibits, like DQ. Thousands of visitors and dozens of school groups each month go through the DQ exhibit as part of their visit to DSC, so that visitors typically go through DQ while other people are doing the same, thus visually perceiving the shared commonality of a DQ walkthrough or play experience.

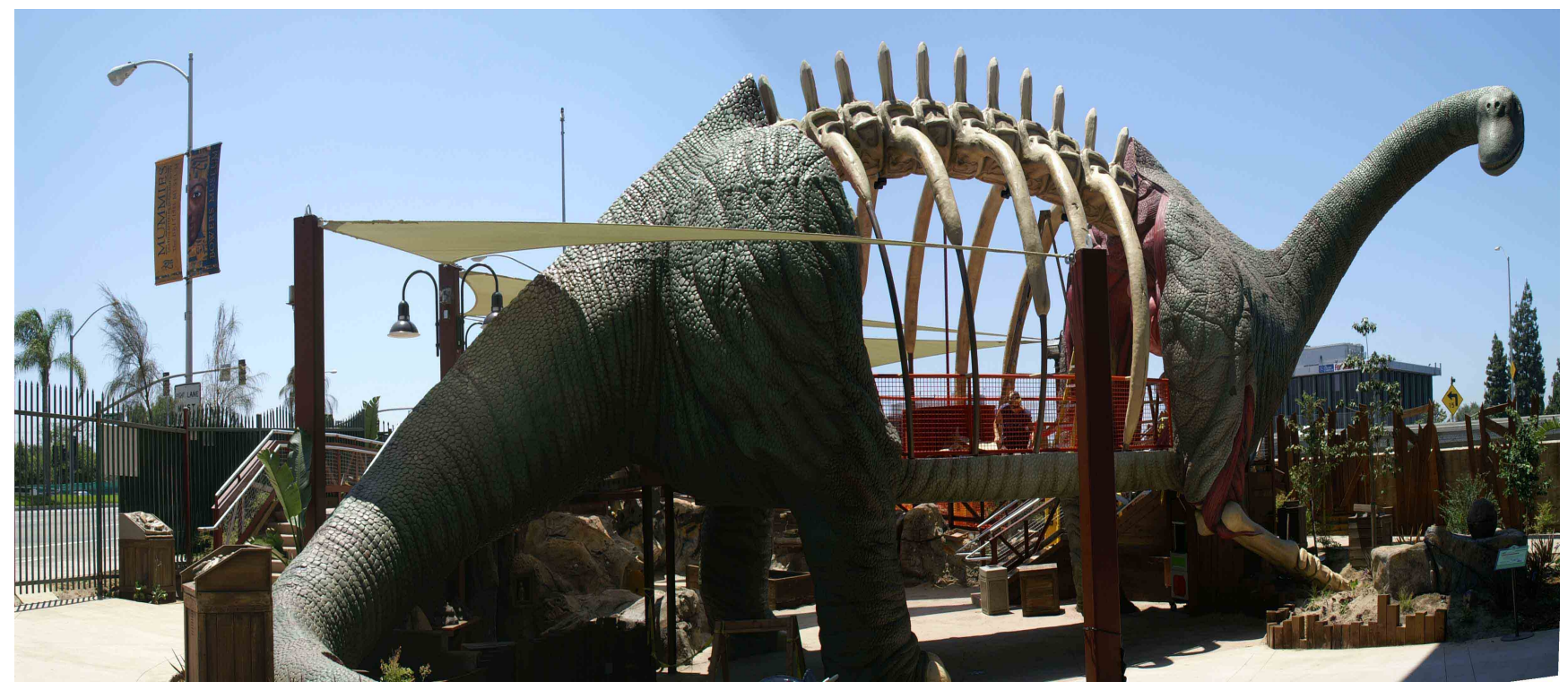

Figure 1. Partial view of DinoQuest with the Argentinosaurus in view

Quest-style (treasure hunt) game design - DQ is modeled as a quest-style game common to many roleplaying games. In a quest, players are given assignments or "missions" to complete, which on completion may earn them rewards of some kind. Given the target audience for DQ is children in K-6 grades, some of these missions are similar in spirit to a "treasure hunt", which then helps set the stage for more advanced missions. In DQ, there are eight missions, organized first into a set of six discovery missions (e.g., locating specified skeletal bones in different dinosaurs), and then two additional more advanced missions. The missions are selected at the "Field Station," which represents an on-site facility

4 As there are no known fossilized remains of the internal organs of nearly all dinosaurs, the mechanical characitures are provided as suggestive analogs to help convey that dinosaurs were living creatures with internal organs whose overall purpose may be similar to that found in humans. 
where other quest related materials can be found (e.g., handouts that further explain the learning objectives of each mission). See Figure 2.

Embedded sensor network and sensor activators - DQ utilizes an embedded sensor network with more than 120 embedded sensor sites that are activated when illuminated with an infra-red designator (enduser wand). The design, installation, and programming of this technology for DQ was provided by Creative Kingdoms Inc. ${ }^{5}$ All designator wands have unique digital identifiers that are bound to aDQ player at the Field Station before first entering the DinoQuest venue. Sensor illumination can then be reconciled with respect to which end-user wand has illuminated it, along with the end-user's current mission/quest. This enables the DQ environment to determine whether the user has designated, found, or otherwise discovered the appropriate life science construct (e.g., eye socket) or paleontological element (triceratops head). Figure 3 displays the IR designator wand for playing DQ, as well as a sample view of two children using their wand during a discovery mission.

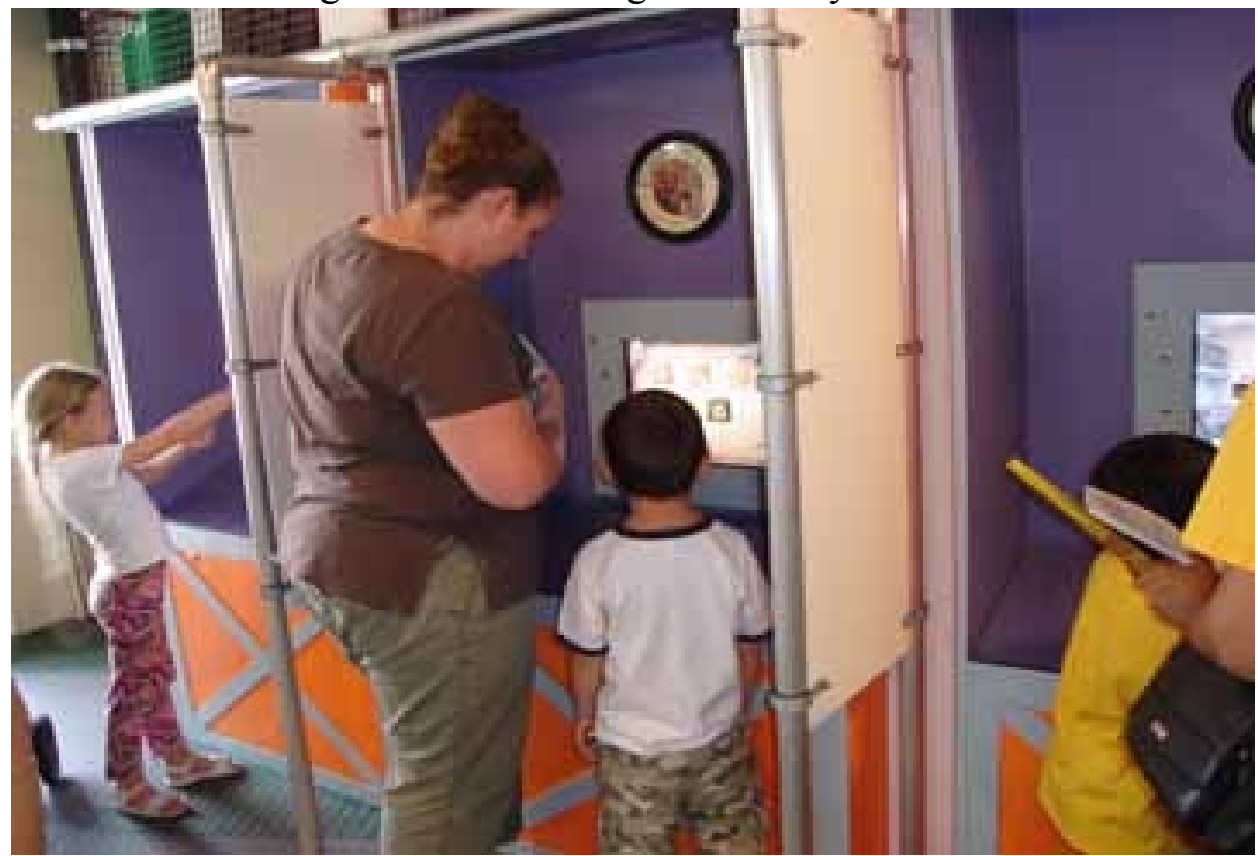

Figure 2. Parent-child team selecting a DQ mission at the Field Station
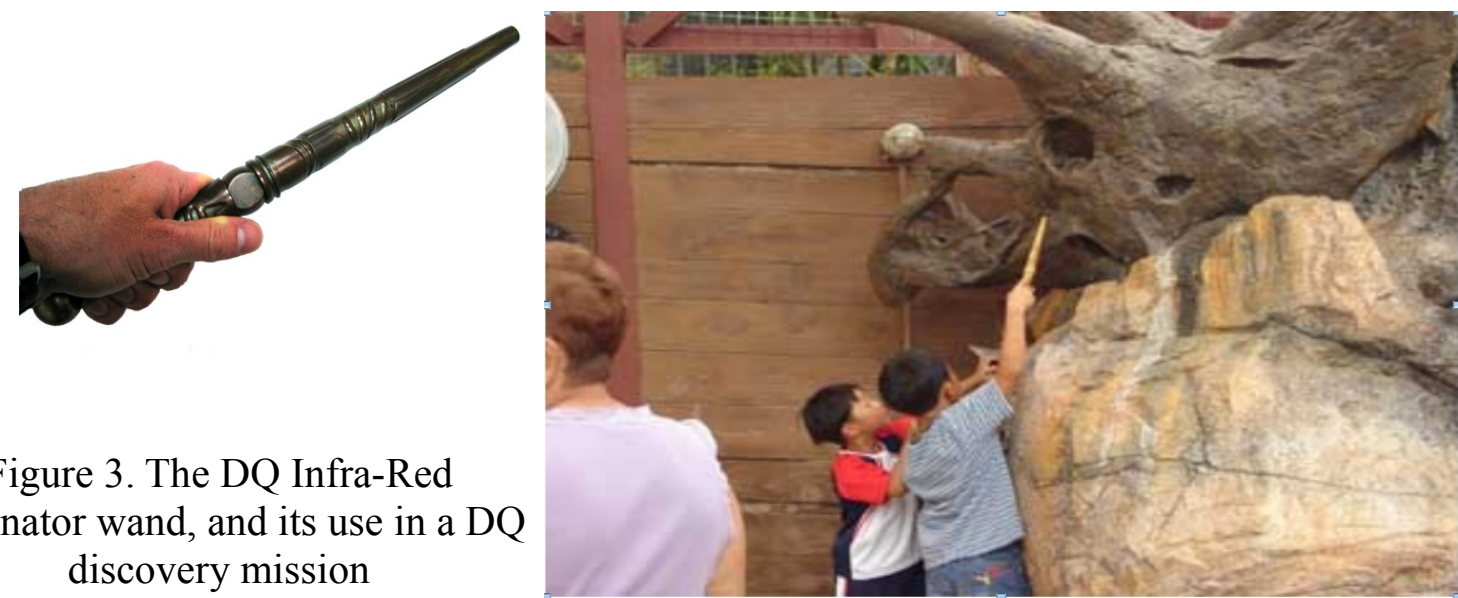

Figure 3. The DQ Infra-Red designator wand, and its use in a DQ discovery mission

5 http://www.creativekingdoms.com/ 
Situated kiosks - Both selection and progress reports associated with each player's current mission can be queried at multi-media kiosks located either in the Field Station or out in the "field." Both selection and progress reporting are triggered by pointing/gesturing the IR wand in the direction of a kiosk.

Kiosks in the field are further assigned as by their association to a virtual collaboratory corresponding to each mission and its geographic location (Africa, Korea, Argentina, United States, etc.). In this regard, we have adopted and integrated the recently developed construct of the research collaboratory [Collabs 2008, Teasley and Wolonsky 2001] into a form that is both (a) accessible to both children, parents, and others in the public, and (b) provides them an introduction, awareness, and simulated experience with such facilities.

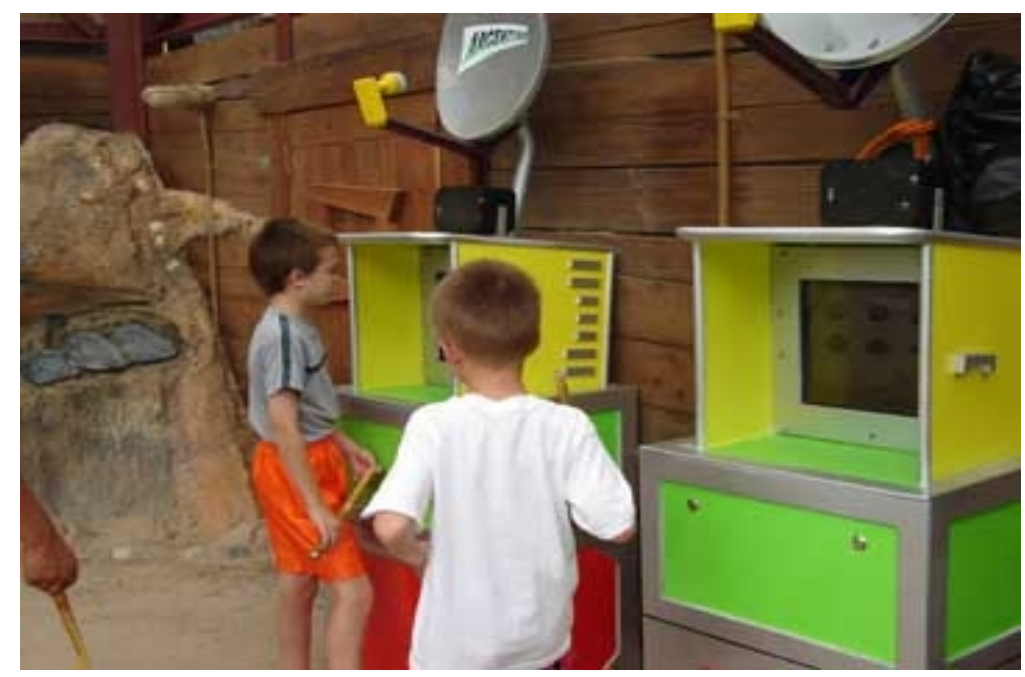

Figure 4. DQ players interacting with kiosks in the field during their current mission

Interactive multi-media presentations on demand-Each kiosk contains a networked personal computer that contains a local cache of pre-recorded multi-media content. These recordings depict people acting in the role of different scientists (e.g., paleontologist, zoologist) in different research settings (paleontology field site, research laboratory) who then interact with DQ players to review or explain features/goals of their missions in progress.
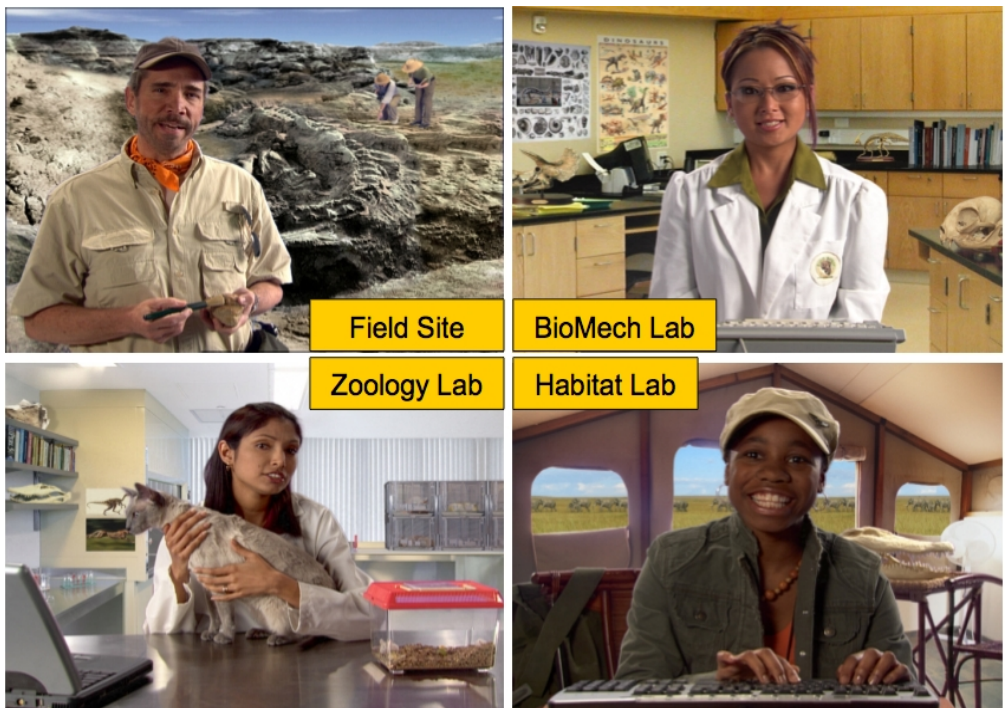

Figure 5. Science role models as DQ collaboratory scientists 
These science role models vary by ethnicity, age, and gender, as shown in Figure 5. The recordings do not reveal answers to the missions until mission completion, but they do provide hints if the player is having trouble completing the mission. As the sensor network monitors each player's discoveries (and misses) and tracks all sensed designation events as transactions to a central database, then when a player queries a kiosk, the system can review their progress to determine which media assets to present to acknowledge progress or to provide some assistance.

Supplementary handout materials for reading and review-The DQ Field Station also is stocked with handout materials. These materials, created by the Education department at DSC, are provided primarily for parents, teachers, and other adults that seek to understand both (a) how to play different DQ missions, and (b) how the missions correspond to California Science Education Standards by school grade level. In general, children players do not consult or review these materials, as it seems they are more comfortable just going out into the DQ venue and playing to discover/learn.

Other DQ visitors - DQ usually is often occupied by dozens of visitors at any time. People in groups (families, school groups) can observe one another and develop a sense about what the others may be doing, and whether these others are having fun, are learning, or seem lost/confused. In particular, we found once DQ became operational that people in different, unrelated groups would at times engage in situated conversation with one another to offer help or advice. Said more simply, people in different groups would collaborate with one another when it seemed they could assist others who might benefit from such assistance. Play in DQ fosters these kinds of situated and emergent collaborations.

Scientific modes of inquiry and field research practices - A central component for developing scientific literacy is developing an understanding for how to engage in scientific modes of inquiry and reasoning. Once again, science education standards include this component at a level appropriate for K-6 grade learners. DQ game play supports various modes of inquiry and field research practice, even within discovery missions. First, DQ missions are thematically organized around topics like identifying trace fossils, and anatomical components and configurations. Second, missions are accumulativerecognizing prey-predator relationships revealed through anatomical features (e.g., small body, long legs, and sharp teeth for meat-eating raptors/T. Rex compared to the large body, short legs, toothless beak for plant eating triceratops). Third, learning (i.e., completing the game) requires observing the environment, gathering facts, and recording or reporting them. Fourth, successful missions can produce results that may be useful later (i.e., reusable) in other missions or problems. Fifth, exhaustive search is not a productive strategy in all situations, and sometimes a search that is focused due to knowledge about features, characteristics, or properties the problem at hand can lead to useful results more quickly. Sixth, scientists come from diverse cultures and may work in distant locations, so someone like you could become a scientist if you have the desire and are willing to do the work required. Last, scientific work entails knowing how to (a) read, communicate, and interact with other people, (b) use tools or instruments, and (c) use telecommunications and information technologies to organize and report data, as well as to communicate and collaborate with others at a distance. Of course, how well DQ players can remember, transfer, and apply such matters in other settings remains an open question.

\section{DinoQuest Online}

The DinoQuest Online venue-The DQO venue is a publicly available Web site that downloads a Flash-based DQO game engine needed to play the game. The DQO game engine dynamically loads the content associated with each game module. DQO currently supports 13 game modules. Each module is a game, and much like DQ, the modules are partially ordered and game play results/knowledge are 
accumulative. The DQO game modules do not mirror the DQ environment or play experience. Instead, DQO provides a set of simulated environment, some literal, other strictly conceptual. Figure 6 provides a view of the in-game home for DQO that appears as a multi-media computing laboratory or collaboratory [cf. Collabs 2008, Teasley and Wolensky 2001]. In the figure, the large multi-panel wall display serves as the in-game interface for "connecting" to remote collaboratories in geographic locations that reflect the same choices in DQ. Selecting one of these collaboratory panels transitions the user the associated game modules. The large map display is the "DinoSphere" which is a higher level, multi-player game space that is accessible only after completing the other game modules. The doors to the right take the user to "MyLab", which is the user's private in-game laboratory office where their research points and other objects collected through both DQ and DQO are kept for later use. The computer screen in the foreground is an interface to an embedded multi-media presentation from an avatar also introduced in DQ. Most of the 13 game modules can be played by a single user, but based on observations at the DSC, children often play DQO with an adult/parent companion who wants to share the game play experience, engage the child player with a discussion about game play, or seek an explanation from the child about what's going on in the game (more often than not, the child needs to explain how the game works to the parent/adult who doesn't usually play computer games).

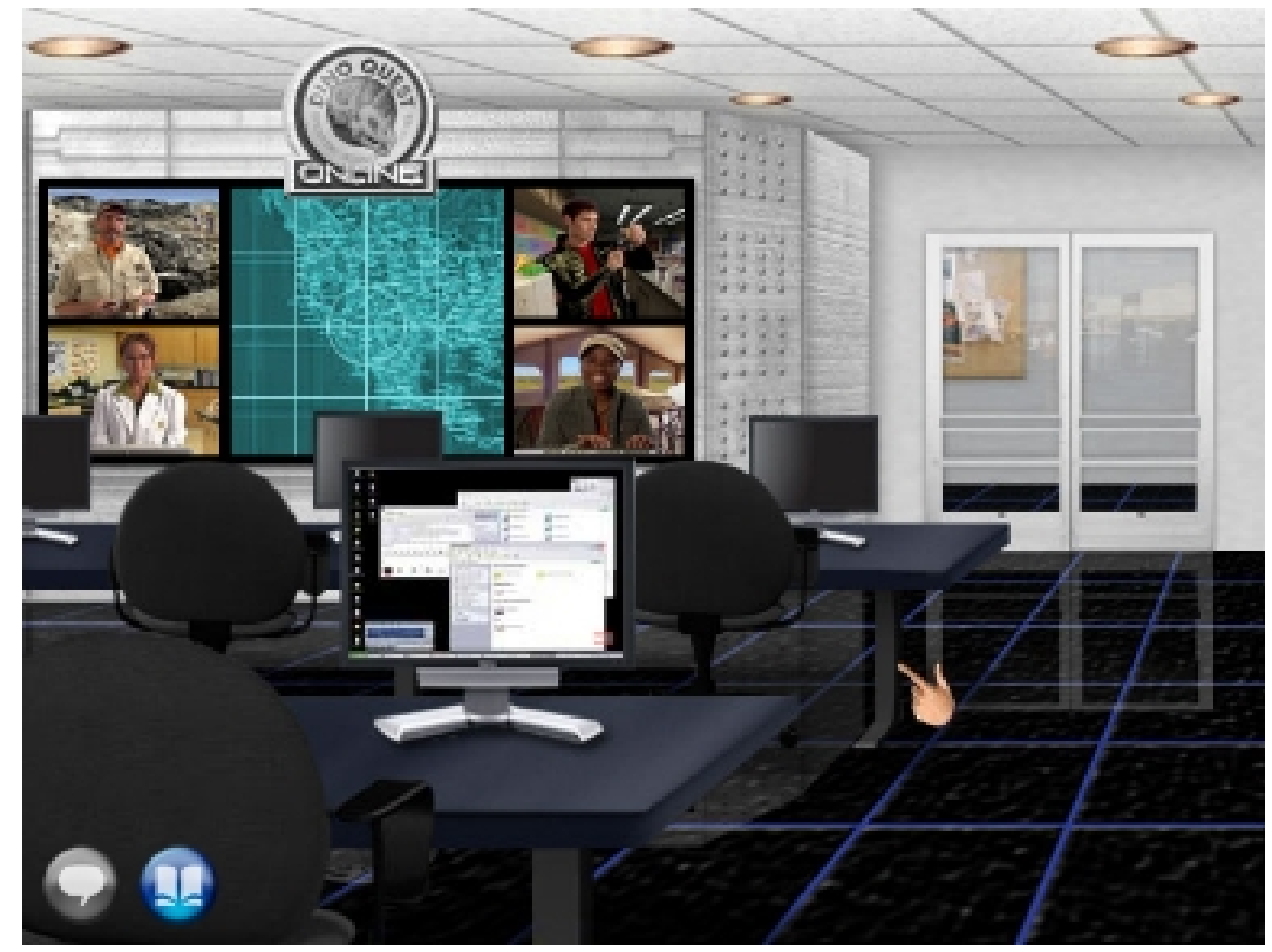

Figure 6. DinoQuest Online in-game home

Multi-genre game design -As previously indicated, DQO consists of 13 game modules. Collectively, they take a player about 3-5 hours to play to completion. However, individual game modules vary in the duration, exposition of life science concepts, and game genre. For example, DQO game modules includes games drawn from quest, design/simulation, puzzle, and mini-game genres. However, it may also be fair to say that these games can individually or collectively be viewed as "casual games" that can be started, played for a brief period, stopped, and restarted later. However, game scores and research points earned persist across game play sessions, as long as the same user (identifier) is 
playing.

Multiple in-game user controls - While DQ benefits from the use of an embedded sensor network and infra-red wand sensor designators, DQO utilizes multiple in-game user controls. For example, as (partially) indicated in Figure 7, the user interface to the fossil dig pit game associated with the Argentine collaboratory employs multiple, task-specific cursors (hand, pick, shovel). The hand is used to select other tools, or to pick up and move (to a storage mechanism) a fossil bone structure that has been "dug up" through game play. The pick is used to only break rocks that appear on the dig pit grid. The shovel is only used to dig into a dig pit grid cell, one at a time. The in-game dashboard on the upper right keeps score of the times used versus available to use in order to self-monitor progress and to create a resource scarcity. These in turn help motivate users to carefully choose when and when to use each user controlled in-game tool.

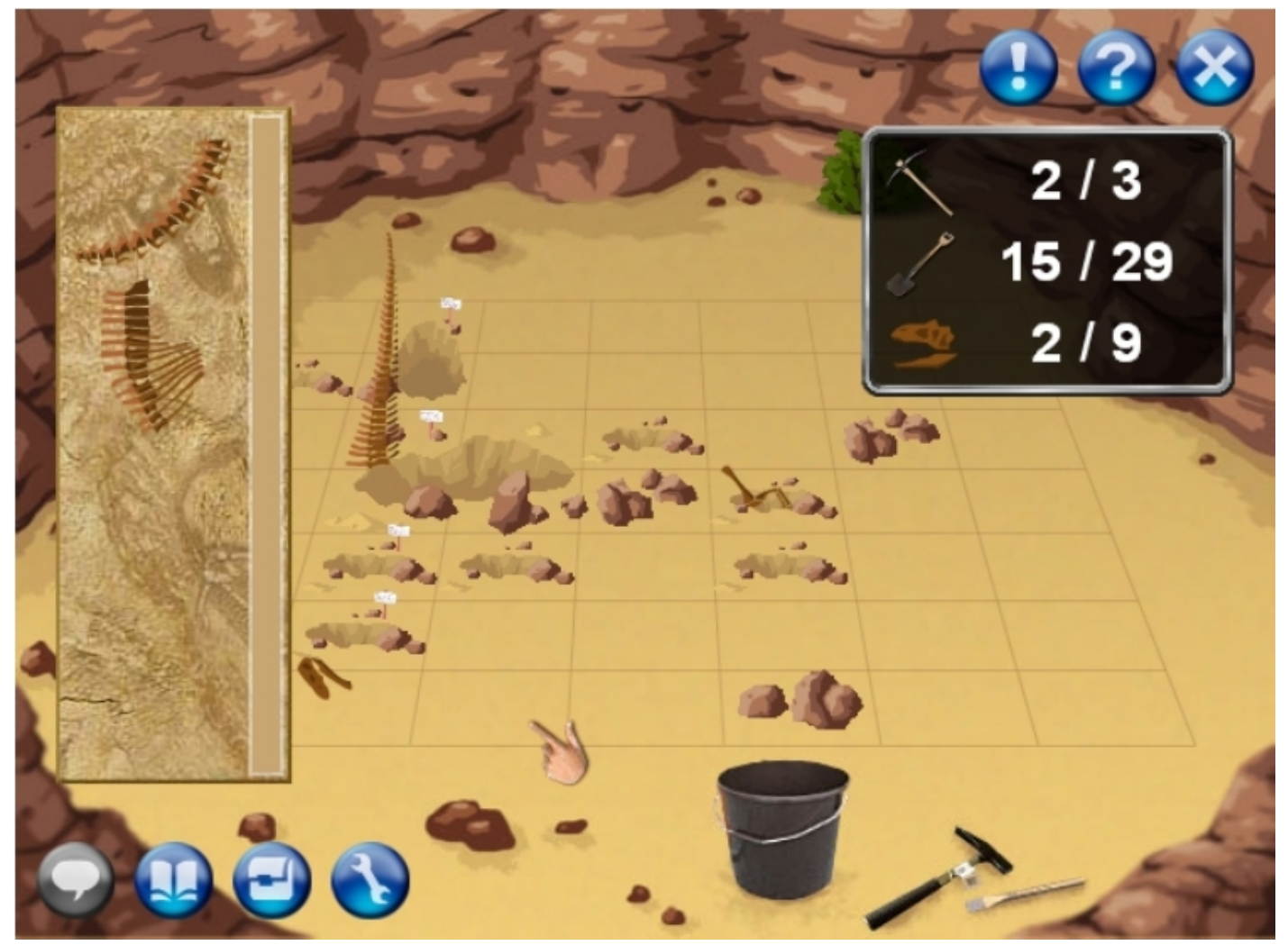

Figure 7. The Fossil Dig Pit game module showing different in-game user controls (hand, pick, shovel) and a dashboard indicating resource utilization (number of possible uses of the pick and shovel).

DQO game environment-DQO represents a contemporary game platform. It is coded in Flash 8, which runs in most commonly available Web browsers (e.g., Internet Explorer, Firefox, Safari). It is accessed from a single Web site (www.DQOnline.org), which in turn downloads the DQO game engine into the user's Web browser, which in turn downloads each game module and its content on user demand. Many families and school groups access and run the DQO game environment on a desktop or laptop computer, as DQO does not require high-end microprocessors, graphics accelerator cards, or the like. Accordingly, the design of the DQO game environment was conceived to enable the largest possible audience of end users or players, including those who may have older, less powerful computers, which includes many under-privileged schools. 
Embedded multi-media content-DQO draws on and shares some of the multi-media assets originally created for presentation on the DQ kiosks. Figure 8 shows an example of an embedded video file that is played on command, and provides a brief explanation of the goals and levels found in DQO. The avatar is the same in-game character that plays the same role in DQ. Use of these in-game characters across DQ and DQO creates a sense of continuity in content and play experience, even though DQ is played in an mixed reality environment, while DQO is play in an online environment.

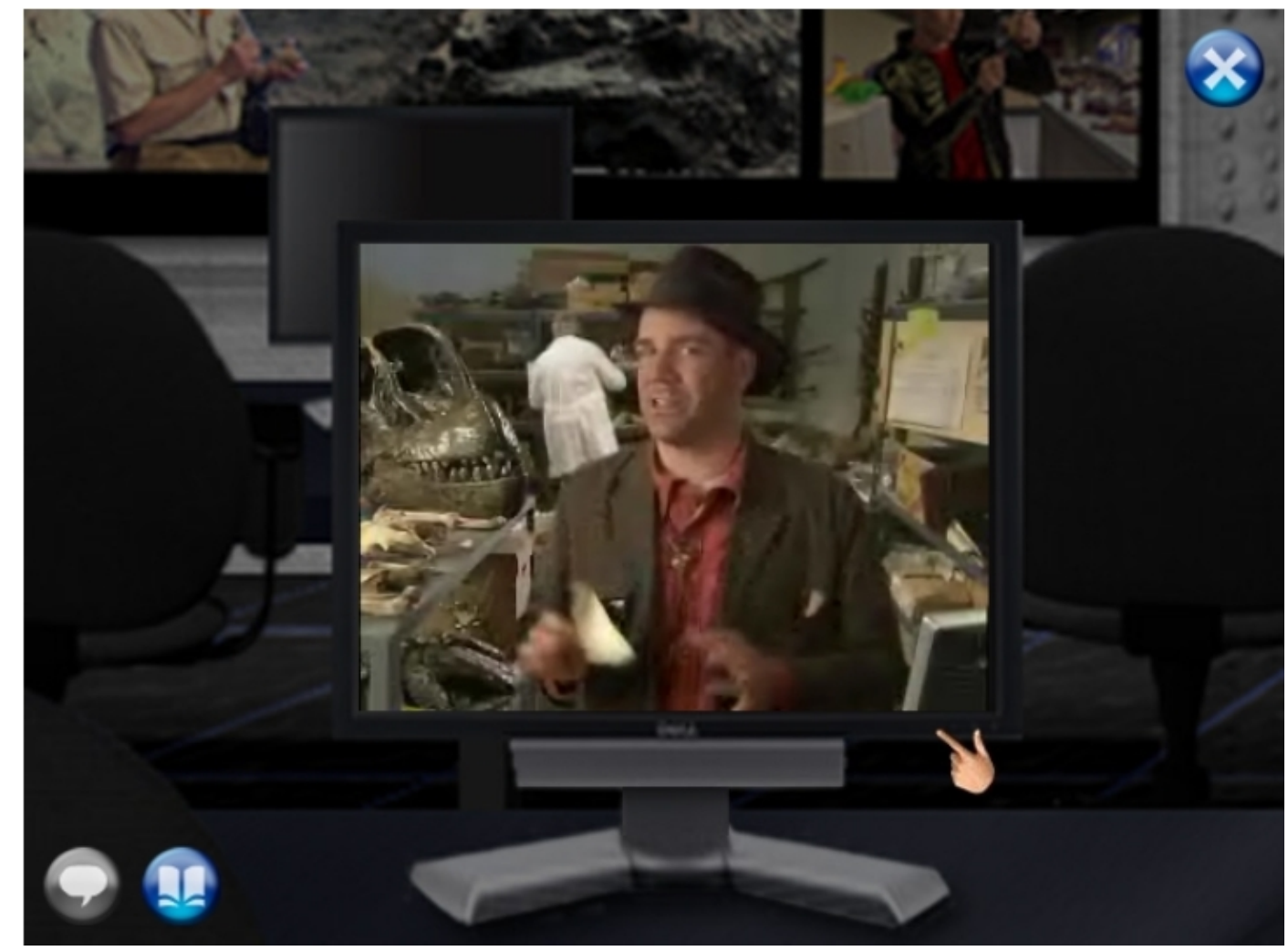

Figure 8. An in-game scene in DQO with an embedded video displaying featuring an in-character also in DQ that introduces and explains DQO's goals and levels.

Embedded tutorials for teachers and parents - It might be surprising to learn that teachers and parents more often than child players want to know how the game operates by reading some prepared materials. Child players on the other hand, often have little/no problem figuring out how to play each DQO game or how to use the in-game user controls, as game play helps to motivate or explain each in a situated way as needed (i.e., children are willing to try something in the game to figure it out, rather than first reading about what to do). Gee [2003] reports that children who learn to play games in such a manner often acquire deep knowledge of the in-game specialist language, terminology, and game play moves that are difficult to determine by a competent adult just by reading a game manual. However, in order to help satisfy the requests from parents, teachers, and other educators, we added a series of embedded tutorials and in-context explanations to help teachers and parents better understand what their children may already know. Figure 9 provides a display of in-game help that is part of such a tutorial for one of the game modules. Beyond this, as DQO players progress from game module to module, DQO also displays interstitial (and stylized) text panels that provide further contextual information about some of the underlying scientific concepts or discoveries that are recreated in the 
game. These interstitial images (or cut scenes) also serve to occupy the player group (e.g., child and parent) with a simple diversion while the next game module in being downloaded and readied for play.

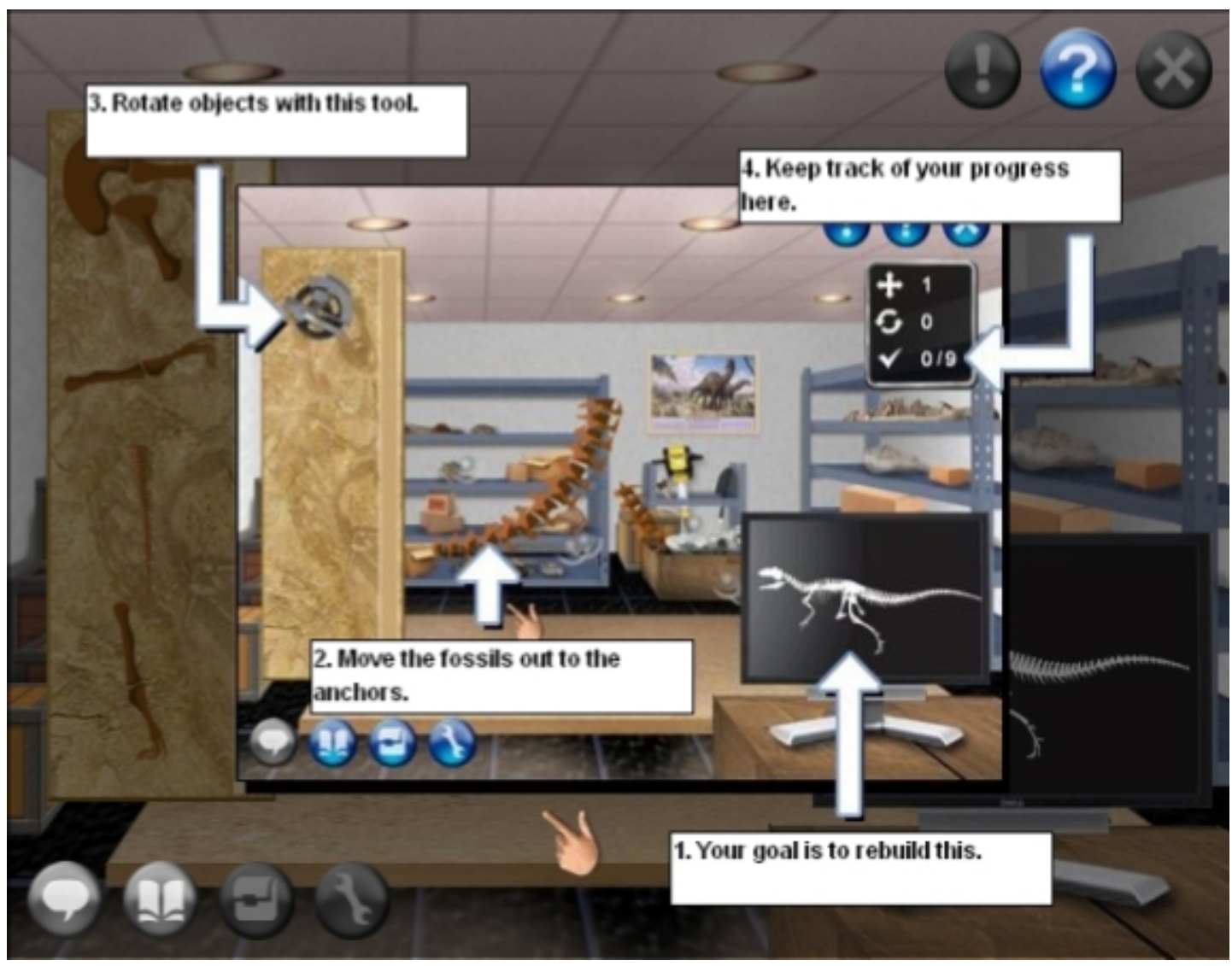

Figure 9. An in-game view of a teacher/parent tutorial explaining the goal and process for playing this DQO game module (reconstructing fossilized skeletal bones collected in the Fossil dig pit module)

Contemporary game play practices used to elucidate life science concepts - SLGs, as games, need to be more than just interactive presentation of scientific concepts, or simulations of scientific practices or processes. As such, we sought to find way to utilize both original and familiar game play mechanisms and play practices in developing each of the DQO game modules. In addition to countless hours we have individually spent playing dozens of games of all sorts, we have found some DQO players can readily recognize game play mechanisms and play practices that we adopted and adapted from other popular games. For example, in Figure 10, we see a view of the ecological relationships game module within DQO that enables play with prey-predator and food chain relationships. This module utilizes a "Tetris" style of game play, where a configuration of ecological elements (carnivores/predators, herbivores/prey, and plants) can be rotated as they move from left to right to match up with configurations that have already been anchored, in order to maximize the matches (e.g., carnivores prey on herbivores, herbivores prey on plants, unmatched carnivores die and help nuture plants). As such, we (and many adult players familiar with Tetris games) find this game is both familiar to play, yet at the same time, presents basic life science concepts by repurposing contemporary game mechanisms and practices. 


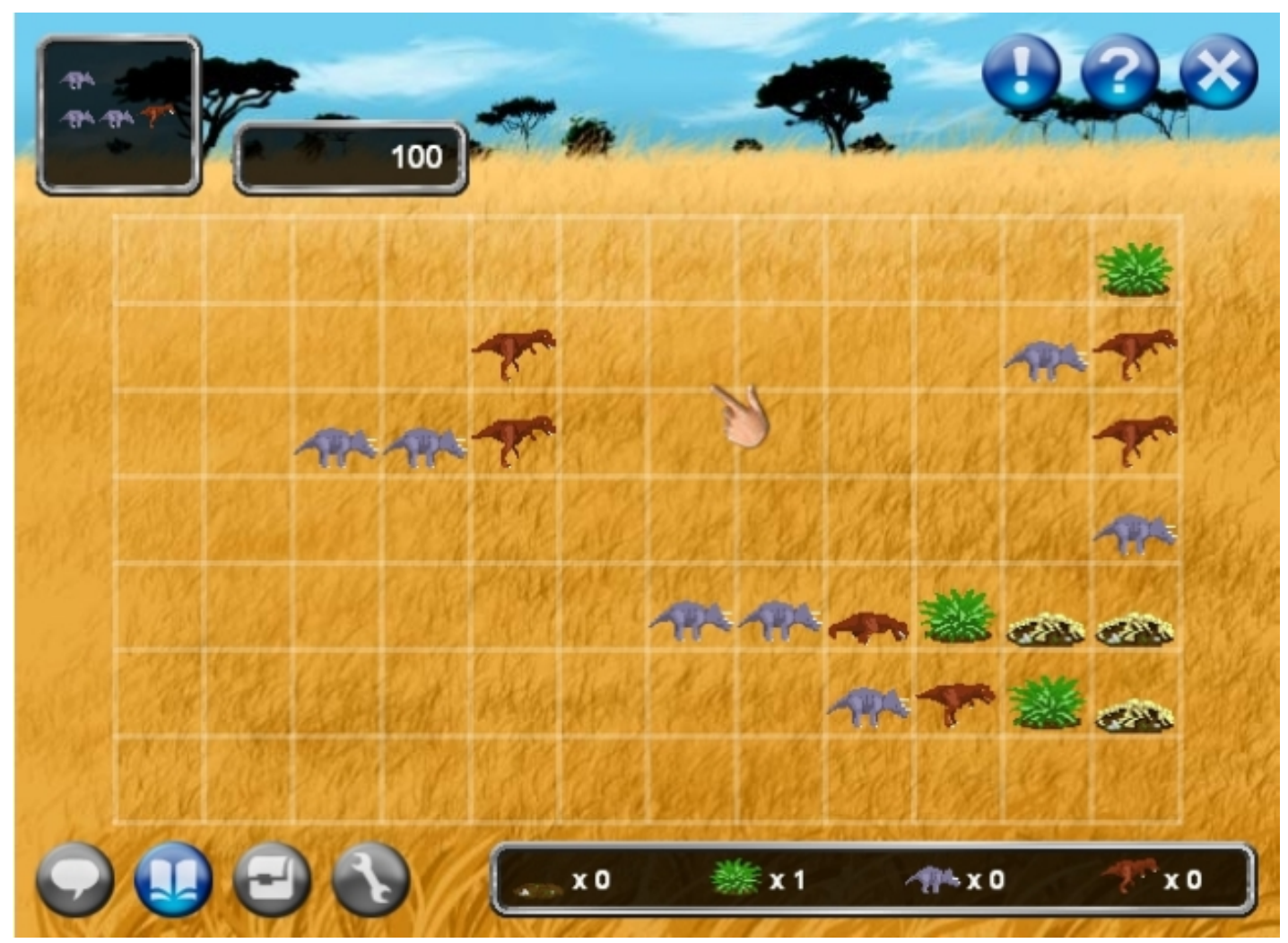

Figure 10. A Tetris-like game for matching ecological relationships like prey-predator and food chains

Multi-person game play-As we have indicated above, it is possible for an individual to play DQO without others. However, it's less fun that way, though it can be quite absorbing and attention grapping for some child players. Instead, when parents/adults want to know what their young children are doing when using a computer accessing information on the Web, they will often join with the child to either play with, share, or engage the player while they play DQO. Beyond this, DQO also features a final level game module, DinoSphere, which is a multi-player or multi-character game module. In this module, player specify and configure a dinosaur of their choosing, using the resources and points they have earned from previous game play. DinoSphere features four ecological niches that serve as simulated physical world environments where different dinosaurs must survive or co-habitate. As players by this point have already learned about life science concepts like prey-predator relationships, then the quickly realize small predators (e.g., raptors) individually are not a threat to larger prey (stegosaurus), unless they can find other similar predators who can then collectively act to surround and overwhelm a larger prey. Figure 11 provides a view of a forest ecological niche within DinoSphere where one small raptor seeks to engage a larger stegosaurus as prey, but without success.

\section{$D Q$ and $D Q O$ together}

Emerging DQ game play experiences that bridge physical and online activity - many visitors to the DSC and DQ come to find that they can continue their (science learning) game play experience online after visiting DQ. On exit from the DQ venue, pathways guide visitors to walk by a group of PCs all configured to run DQO. Visual signs provide encouragement to sit down and try out DQO on the spot, while other guidance describes how to continue to play DQO at home over the Web at http://www.DQOnline.org. Frequently, DQ visitors who have already gone through DQ grab a seat in front of an available PC and start to play and explore DQO. Other DQ visitors in turn observe the DQO 


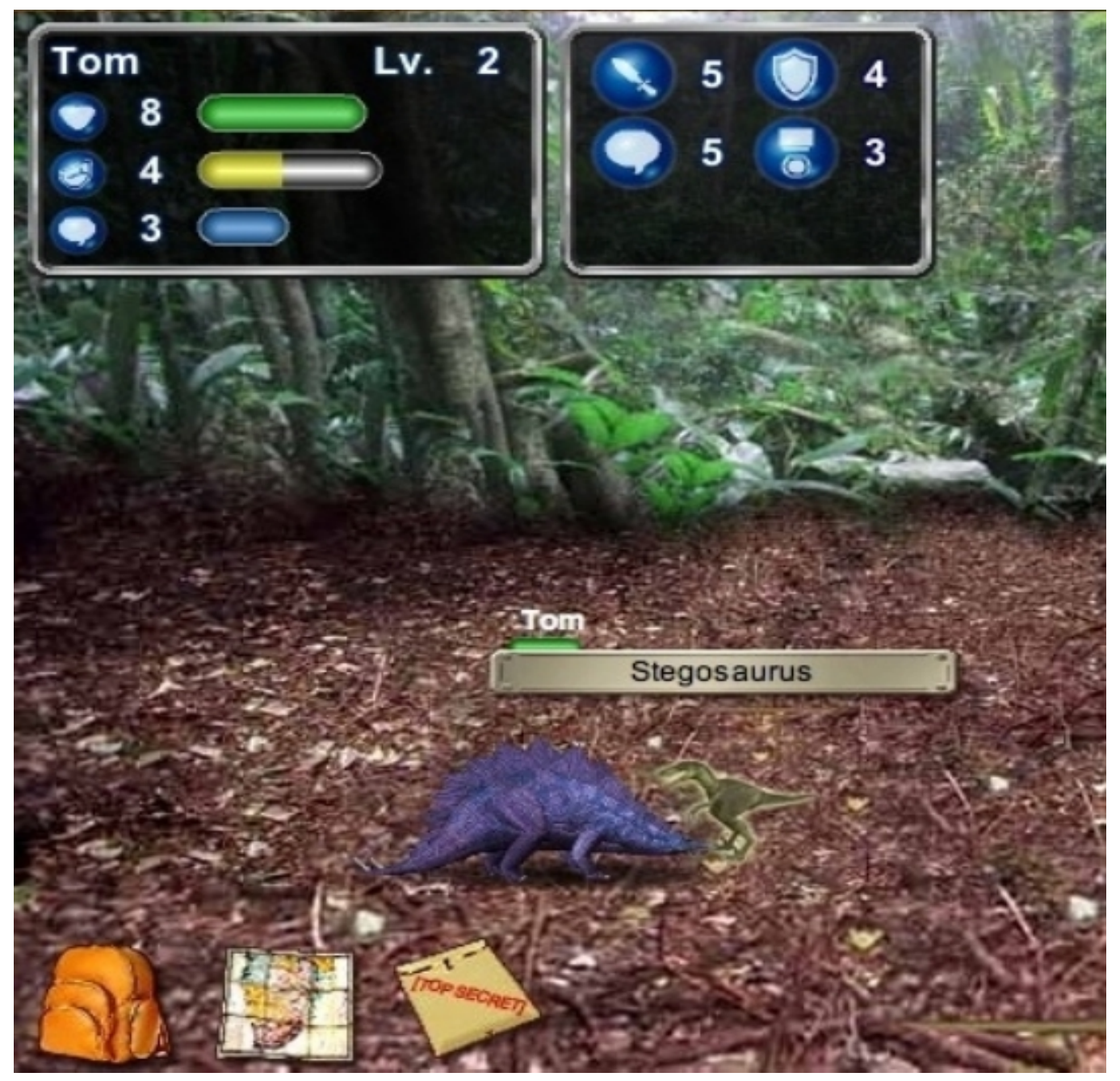

Figure 11. A scene from the DQO module, DinoSphere, where multiple players or in-game characters (e.g., stegosaurus and raptor) can interact in a simulated ecological niche to survive or thrive. The dashboard at the top displays the status of various resources controlled by the player Tom.

game play in progress, and this often helps interested and Web-savvy DQ visitors to note how to later access DQO for further SLG play at home with family or friends.

Interoperability linking between $D Q$ and $D Q O-\mathrm{DQ}$ and $\mathrm{DQO}$ were co-designed from the beginning to work together. How they were intended to work together did change throughout the development process. This may not be surprising given that we were looking at alternative ways to link both the games and the game play experiences together. The engagement of Creative Kingdoms Inc. help to provide a strategy and technical infrastructure for how to connect the physical game of DQ with the online game play of DQO. As both DQ and DQO would be based on databases, it was then possible to develop a system (server) architecture and data sharing regime whereby user registrations and scores/points could be exchanged between the DQ and DQO databases, as well as providing a security mechanisms (firewall and data replication server) so that either game system could operate, if needed, without the other, but otherwise share common data in a controlled and tractable manner. Finally, it should be noted that another reason for linking DQ and DQO together was to also create in-game 
incentives for players (e.g., families) who access DQO over the Web to come to the DSC to complete their game play - or more simply, to help increase visitor attendance and membership at DSC. As a result (or maybe as just a coincidence) attendance at DSC has grown significantly since DQ and DQO became operational. This means more families and school groups are coming to DSC, and they are repeatedly visiting DSC at a much higher rate than before DQ and DQO were available.

\section{Discussion and planned enhancements to $D Q$ and $D Q O$}

The first topic of discussion is whether DQ and DQO are fun to play. Since fun may be in the mind of the player, we can report results from sustained informal observations (along with a multitude of DSC administered surveys and interviews) that the quick and simple answer is: yes, they are fun to play, but each is fun in a different way. Second, do the players learn anything useful about scientific inquiry or life science? Again based on the same instrumentality for observation, the quick and simple answer is yes, though what is learned in DQ and DQO are different. In both situations, DQ is a game played in a physical venue with other people and involves personal activity and mobility. DQO is a game played in an online, Web-based venue where other people may be involved in play, but their involvement may be centered around interaction at the human-computer interface or through in-game dinosaur characters foraging in a simulated ecological niche. So we expect that what's fun and what's learned will be different, but we continue to seek to understand how and why they are different.

The physical DQ venue and game play experience is difficult to upgrade, while the DQO venue (at least the game engine software, game content, and back-end database services) can be more readily upgraded, as resources become available. Accordingly, we are investigating a number of planned upgrades to DQO that will further expand the collaboration affordances and game play experiences that can be realized more readily in an online, Web-based game platform.

First, reflecting the diversity of people (students, parents, and others) who visit DSC, we seek to provide multi-lingual game play user interfaces for DQO in languages such as Spanish and Korean. Actually, we developed DQO with internationalization and localization as part of its design and implementation scheme, so provision of multi-lingual support is primarily one of adding/replacing corresponding in-game textual content across languages.

Second, following from this, we seek to provide multi-national deployments for DQO to non-English speaking venues. Dinosaur themed interactive science exhibits are being developed in areas like Mexico, Latin America, and South Korea. Though DQO was designed with California Science Education Standards in mind, our correspondence with colleagues in those areas indicates such standards are acceptable as a starting point.

Third, we seek to expand the multi-player capabilities of the DQO DinoSphere to accommodate more MMOG services and game play modes. Our intent is to add such capabilities to the highest levels of DQO DinoSphere game play, so that existing game content and play experience will be minimally affected. The wisdom of this choice remains to be seen and realized.

Last, our original goals included making DQO a networked SLG environment that could be built from open source software, support open content artwork, and accommodate a controlled interface to the Web of open and current scientific research related to paleontology and paleobiology. None of this has been realized, and the technical choices that we have invested will limit what we can do to realize these 
goals. Nonetheless, they remain part of our long-term goal, and we look forward to opportunities that will enable to develop more collaborative SLGs in other scientific domains and for other public audiences who want informal science education experiences and resources.

\section{Conclusions}

In this paper, we described some of the concepts and results that arose from the development and deployment of a large-scale collaborative game environment called DinoQuest and DinoQuest Online. As an interoperable game environment, DQ and DQO provide a unique experience and approach to informal science education, as well as matching California/National Science Education Standards. As noted, games for helping school-age children to learn about science (or more specifically, life science) is not a focus of the global computer game industry, yet we believe it represents an important and under-served community of potential game players and others (parents, teachers) who want to informally collaborate in and around such a science learning game environment. Subsequently, we identified and examined different collaborative group forms that emerged to play DQ and DQO, as well as the affordances that help facilitate collaborative game play. Along the way we provided examples of the collaborative groups, affordances, and game play from DQ and DQO.

In closing, we welcome readers to either make their way to Santa Ana to visit the Discovery Science Center to see and experience DinoQuest, or to register and play one or more of the DinoQuest Online game modules found starting at http://www.DQOnline.org. Both DQ and DQO will provide different but comparable experiences for collaborative games and game play, each of which can last for 3-5 hours.

Acknowledgements: Development of DinoQuest and DinoQuest Online was supported by the Discovery Science Center, its members, and many governmental and corporate sponsors. Recent R\&D projects at the UCGameLab are supported with contracts and grants from the Digital Industry Promotion Agency (DIP) in Daegu, South Korea, Intel Research, California Institute for Telecommunications and Information Technology (Calit2), and others. No endorsement implied. Some of the other participants involved in the development of DinoQuest and DinoQuest Online include Creative Kingdoms, Inc., Alex Szeto (DQO game art and programming), Calvin Lee (DQO database programming), Janet Yamaguchi (California Science Education Standards, and Education Programs at DSC), and Celia Pearce (design contributions).

\section{References}

Allison-Bunnell, S. and Schaller, D.T. (2005). From the Physical to the Virtual: Bringing Free-Choice Science Education Online, in L. Tan and R. Subramanian (eds.), E-Learning and Virtual Science Centers, 163-189, Hershey, PA, IGI Global Publishing, 2005.

Anderson, R. and Sharrock, W. (1993). Can Organisations Afford Knowledge?, Computer Supported Cooperative Work, 1(2), 143-161.

Bainbridge, W.S. (2007). The Scientific Research Potential of Virtual Worlds, Science, 317, 472-476.

Bogost, I. (2007). Persuasive Games: The Expressive Power of Videogames, MIT Press, Cambridge, MA. 
Collabs, (2008). The Science of Collaboratories, http://www.scienceofcollaboratories.org/

DSC, (2008). Discovery Science Center, Santa Ana, CA. http://www/discoverycube.org

Gee, J.P. (2003). What Computer Games Have To Teach Us About Learning and Literacy, Palgrave Macmillan, New York.

Koster, R. (2004). A Theory of Fun for Game Design, Paraglyph Press.

LaFarge, A. and Nideffer, R. (2002). Shift-Ctrl: Computer, Games, and Art, Leonardo: A Journal of Art, Science, and Technology. MIT Press. 35(1), 5-13, Winter.

Malone, T. and Lepper, M.R. (1987). Making Learning Fun: A taxonomy of intrinsic motivations for learning, in R.E. Snow and M.J. Farr, Aptitude, Learning, and Instruction III: Conative and Affective Process Analyses, 223-253, Erlbaum, Hillsdale, NJ.

Muramatsu, J. and Ackerman, M.S. (1998). Computing, Social Activity, and Entertainment: A Field Study of a Game MUD, Computer Supported Cooperative Work, 7(1), 87-122.

Nardi, B. and Harris, J. (2006). Strangers and Friends: Collaborative Play in World of Warcraft, Proc. 2006 Conf. Computer Support Cooperative Work (CSCW'06), 149-158, Banff, Canada, ACM Press.

Nideffer, R. (2002). PROXY, The Whitney Museum Portal to Net Art, http://artport.whitney.org/exhibitions/biennial2002/nideffer.shtml.

Nideffer, R. (2006). unexceptional.net, http://transliteracies.english.ucsb.edu/post/researchproject/project-members/robert-nideffer

Nideffer, R. (2007). Game Engines as Embedded Systems, in V. Vesna (ed.), Database Aesthetics: Art in the Age of Information Overflow. University of Minnesota Press. Minneapolis, Minnesota.

O'Day, V., Bobrow, D., Bobrow, K., Shirley, M., Hughes, B., and Walters, J. (1998). Moving Practice: From Classrooms to MOO Rooms, Computer Supported Cooperative Work, 7(1), 9-45.

Rieber, L. (2005). Multimedia learning in games, simulations, and microworlds. In R. E. Mayer (Ed.), The Cambridge Handbook of Multimedia Learning (pp. 549-567). New York: Cambridge University Press.

Salen, K. and Zimmerman, E. (2003). Rules of Play: Game Design Fundamentals, MIT Press.

Scacchi, W. (2004). Free/Open Source Software Development Practices in the Computer Game Community, IEEE Software, 21(1), 59-67, January/February 2004.

Scacchi, W. and Adams, J. (2007). Recent Developments in Science Learning Games for Informal Science Education, presentation at Games, Learning, and Society: 3.0, Madison, WI. July.

Shaffer, D. W. (2006). How Computer Games Help Children Learn, Palgrave Macmillan, New York. 
Teasley, S., \& Wolensky, S. (2001) Scientific collaborations at a distance. Science, 292, 2254-2255.

UCGameLab, (2008). Game Culture and Technology Laboratory, University of California, Irvine, Irvine, CA. http://www.UCGameLab.net

WDIL (2008). Web Designs for Interactive Learning, http://www.wdil.org/ 